\title{
Exposure and CBT for Chronic Back Pain: An RCT on Differential Efficacy and Optimal Length of Treatment
}

Citation for published version (APA):

Glombiewski, J. A., Holzapfel, S., Riecke, J., Vlaeyen, J. W. S., de Jong, J., Lemmer, G., \& Rief, W. (2018). Exposure and CBT for Chronic Back Pain: An RCT on Differential Efficacy and Optimal Length of Treatment. Journal of Consulting and Clinical Psychology, 86(6), 533-545.

https://doi.org/10.1037/ccp0000298

Document status and date:

Published: 01/06/2018

DOI:

$10.1037 / \mathrm{ccp} 0000298$

Document Version:

Publisher's PDF, also known as Version of record

Document license:

Taverne

Please check the document version of this publication:

- A submitted manuscript is the version of the article upon submission and before peer-review. There can be important differences between the submitted version and the official published version of record.

People interested in the research are advised to contact the author for the final version of the publication, or visit the DOI to the publisher's website.

- The final author version and the galley proof are versions of the publication after peer review.

- The final published version features the final layout of the paper including the volume, issue and page numbers.

Link to publication

\footnotetext{
General rights rights.

- You may freely distribute the URL identifying the publication in the public portal. please follow below link for the End User Agreement:

www.umlib.nl/taverne-license

Take down policy

If you believe that this document breaches copyright please contact us at:

repository@maastrichtuniversity.nl

providing details and we will investigate your claim.
}

Copyright and moral rights for the publications made accessible in the public portal are retained by the authors and/or other copyright owners and it is a condition of accessing publications that users recognise and abide by the legal requirements associated with these

- Users may download and print one copy of any publication from the public portal for the purpose of private study or research.

- You may not further distribute the material or use it for any profit-making activity or commercial gain

If the publication is distributed under the terms of Article $25 \mathrm{fa}$ of the Dutch Copyright Act, indicated by the "Taverne" license above, 


\title{
Exposure and CBT for Chronic Back Pain: An RCT on Differential Efficacy and Optimal Length of Treatment
}

\author{
Julia Anna Glombiewski, Sebastian Holzapfel, \\ and Jenny Riecke \\ Philipps-University Marburg \\ Jeroen de Jong \\ Maastricht University
}

\author{
Johan W. S. Vlaeyen \\ University of Leuven and Maastricht University
}

\begin{abstract}
Objective: Our aim was to establish whether Exposure, a specialized tailored treatment for chronic low back pain, has any advantages over cognitive-behavioral therapy (CBT) among individuals with high fear-avoidance levels. Second, we planned to compare short and long versions of Exposure. Third, we aimed to investigate whether Exposure can be delivered in an outpatient psychological setting. Method: A total of 88 Caucasian participants (55\% women) were randomized to three different psychological treatment conditions, Exposure-long, Exposure-short, and CBT. All participants were suffering from chronic pain and elevated levels of pain-related anxiety and disability. The primary outcomes were disability (assessed using two different questionnaires, QBPDS and PDI) and average pain intensity; secondary outcomes included pain-related anxiety, psychological flexibility, coping strategies, and depression. Assessments took place at pretreatment, midtreatment, posttreatment, and 6-month followup. Results: Exposure was more effective than CBT at reducing movement-related disability assessed with the QBPDS. Exposure and CBT did not differ in reduction of pain intensity or disability assessed using the PDI. Exposure-short outperformed Exposure-long after 10 sessions, meaning that individuals improved faster when they were offered fewer sessions. Exposure could be safely delivered in the psychological setting. Concerning secondary outcomes, Exposure led to greater improvements in psychological flexibility relative to CBT. CBT was more effective than Exposure at enhancing coping strategies. In Exposure, significantly more participants dropped out. Conclusions: Although being more challenging to patients, Exposure is an effective treatment, which can be delivered in a psychological treatment setting and should be offered as a short-term treatment.
\end{abstract}

\section{What is the public health significance of this article?}

The study suggests that Exposure is an effective treatment of disability in chronic low back pain. Five sessions of Exposure were as effective as ten sessions; therefore, Exposure is especially useful for treating pain patients in multidisciplinary treatments with very limited time and resources for psychological interventions.

Keywords: exposure, CBT for pain, chronic low back pain, fear-avoidance model

Supplemental materials: http://dx.doi.org/10.1037/ccp0000298.supp

Chronic low back pain (CLBP) is a highly prevalent health problem in Western societies. In Europe, one out of every five people experiences significant back pain that interferes with qual- ity of life (Breivik, Collett, Ventafridda, Cohen, \& Gallacher, 2006). CLBP is one of the major causes of medical expenses, work absenteeism, and disability (Van Tulder \& Koes, 2006). Recent
Julia Anna Glombiewski, Sebastian Holzapfel, and Jenny Riecke, Department for Clinical Psychology and Psychotherapy, Philipps-University Marburg; Johan W. S. Vlaeyen, Research Group Health Psychology, University of Leuven and Research Group Experimental Health Psychology, Maastricht University; Jeroen de Jong, Research Group Behavioral Medicine, Maastricht University; Gunnar Lemmer, Department for Psychology, Psychological Methods, Philipps-University Marburg; Winfried
Rief, Department for Clinical Psychology and Psychotherapy, PhilippsUniversity Marburg.

Correspondence concerning this article should be addressed to Julia Anna Glombiewski, who is now at the Department for Clinical Psychology and Psychotherapy, University of Koblenz-Landau, Ostbahnstr. 10, 76829 Landau, Germany. E-mail: glombiewski@uni-landau de 
international guidelines recommend psychological interventions for CLBP over pharmacological treatments (Qaseem, Wilt, McLean, \& Forciea, 2017). Cognitive-behavioral therapy (CBT) is a type of psychological treatment that has been recommended for CLBP (Morley, Eccleston, \& Williams, 1999; Van Tulder \& Koes, 2006). The definition of CBT for chronic pain offered by Turk (Turk, 2003) includes three types of interventions: cognitive (education, cognitive restructuring, and attention shifting), respondent (e.g., relaxation), and behavioral (e.g., activity pacing). The major goal of CBT is to teach different coping strategies for dealing with pain. CBT is a "broad-spectrum" treatment, suitable for the majority of individuals suffering from chronic pain, and is the most common psychological treatment in multidisciplinary settings such as pain clinics and rehabilitation centers. However, the effects of CBT on pain intensity and pain-related outcomes are moderate, leaving room for improvement. In particular, CBT has negligible effects on disability and functioning (Chou et al., 2017).

Thus, there has been a call for more focused, tailored chronic pain treatments specifically addressing subgroups of pain patients in hope of achieving better results (Hasenbring \& Verbunt, 2010). To date, however, there is no empirical evidence as to whether pain interventions specifically tailored for particular subgroups are in fact more effective than traditional, broad-spectrum CBT.

Sixteen years ago, Vlaeyen and colleagues developed a new treatment, graded in vivo exposure for CLBP (Exposure), focusing on a subgroup of individuals with CLBP who avoid activity due to fear of harm (J. W. Vlaeyen, de Jong, Geilen, Heuts, \& van Breukelen, 2001). The theoretical basis for this treatment is the fear-avoidance model of chronic pain, which postulates that fear and avoidance of movement contribute to the maintenance of pain and disability through classical conditioning (J. W. Vlaeyen, Crombez, \& Linton, 2009). The so-called "fear-avoidance" is one of the most important predictors of transition from acute to chronic pain and of pain-related disability (Linton, 2000). Approximately $50 \%$ of individuals suffering from CLBP have elevated levels of fear-avoidance (Linton, 2000), and thus might benefit from a treatment specifically addressing pain-related fear. As in the treatment of anxiety disorders, the patient is gradually exposed in vivo to feared stimuli. For CLBP, these stimuli are different movements believed to lead to pain or potential back injuries. The primary goal of Exposure is to address avoidance in order to reduce pain-related disability over the long term (Boersma et al., 2004).

The evidence on Exposure for CLBP is sparse (Macedo, Smeets, Maher, Latimer, \& McAuley, 2010). Approximately 10 years ago, first single case designs showed that graded Exposure reduced pain disability and pain-related fears with large effect sizes (Boersma et al., 2004; J. W. Vlaeyen et al., 2001). Following the case studies, four randomized controlled trials (RCTs) compared graded Exposure with a waiting list and graded activity programs (Goossens et al., 2015; Leeuw et al., 2008; Woods \& Asmundson, 2008), or standard medical care (Linton et al., 2008). These studies found some benefits of Exposure, including greater reduction in painrelated fear and perceived harmfulness of physical activity, but found no between-groups differences on the primary outcome, pain-related disability. In the physical therapy field, $\mathrm{O}^{\prime}$ Sullivan and colleagues also developed a treatment that focuses on fear of movement, known as "cognitive functional therapy." In an RCT and several case studies (O'Sullivan, Dankaerts, O'Sullivan, \& O’Sullivan, 2015; Vibe Fersum, O’Sullivan, Skouen, Smith, \&
Kvåle, 2013), cognitive functional therapy had superior outcomes for CLBP compared with manual therapy and exercise. The goals of cognitive functional therapy are similar to those of Exposure and graded activity: correcting dysfunctional beliefs about pain and movement, goal setting, and changing movement behaviors using a graded approach (O'Sullivan et al., 2015). The major difference is that cognitive functional therapy was designed to be delivered by physiotherapists and thus includes more physiotherapeutic elements.

Thus, RCTs comparing Exposure to other treatments for CLBP have yielded inconclusive results, with little evidence for effects on pain-related disability. With exception of some multidisciplinary research institutions such as Maastricht University Hospital (den Hollander et al., 2016), Exposure for CLBP is not yet integrated into standard care, and little is known about optimal setting characteristics or other factors facilitating or hindering the successful delivery of Exposure.

In recent years, psychotherapy outcome research has addressed questions of optimal psychotherapy dosage using survival analyses in larger, often routine-care samples or using meta-analyses, merging samples with different psychological disorders and different psychological treatment approaches. Based on these studies, Hansen and colleagues for example, found that between 13 and 18 sessions of therapy are required for $50 \%$ of patients to improve (Hansen, Lambert, \& Forman, 2002), while Harnett and colleagues (Harnett, O'Donovan, \& Lambert, 2010) estimated that it takes 8 sessions for $50 \%$ of clients to show reliable improvement and 21 sessions for $85 \%$ to meet this criterion. These results mainly apply to treatments that last as long as needed. Interestingly, it has also been found that individuals recover faster when they are offered fewer sessions (Barkham et al., 2006). It is unclear whether these results are generalizable to psychotherapy for CLBP, and thus further research is needed on the optimal dose of psychotherapy in chronic pain.

In summary, several questions concerning Exposure treatment for CLBP remain unanswered.

First, since Exposure for CLBP has not yet been compared with CBT, it is unclear whether a specialized CLBP treatment has any advantages over broad-spectrum psychological therapy among individuals with high levels of pain-related anxiety, particularly with respect to reducing disability and improving function. In addition, the optimal setting and length of Exposure treatment have not been established.

In the current study, we compared Exposure and CBT among individuals with CLBP and elevated levels of pain-related anxiety using several outcome measures and assessment time points. We compared two different doses of Exposure (15 sessions, including 10 Exposures), vs. 10 sessions, including 5 Exposures). Although Exposure was originally designed for multidisciplinary inpatient settings, the current study took place in an outpatient setting, and the intervention was implemented by psychologists with a special training in psychotherapy.

We hypothesized that Exposure would outperform CBT at reducing disability due to its focus on avoidance of movements (Hypothesis 1). Based on previous results from single case studies and observations made by Barkham et al. (2006), we expected that 5 sessions of Exposure would (a) be sufficient to achieve satisfactory results and (b) lead to faster improvements compared with the longer treatments (Hypothesis 2). Since we investigated a rela- 
tively new treatment in a specific setting, we placed a high value on assessing treatment fidelity, treatment credibility, side effects, and treatment dropout.

\section{Method}

The study was approved by the Ethics committee of the University of Marburg. The ClinicalTrials.gov Identifier is NCT01484418. This study was supported by a Grant GL 607/5-1 from the German Research Foundation (DFG) to Julia Glombiewski. The treatment protocol has been previously published (Riecke, Holzapfel, Rief, \& Glombiewski, 2013).

\section{Participants}

The study was conducted at the Outpatient Center for Psychological Interventions in the Department of Psychology at the University of Marburg, Germany. The study was advertised via local newspaper articles, advertisements, flyers, and outreach to local hospitals, GPs, and anesthesiologists. Basic inclusion criteria (age 18-66 years, pain in the lower back longer than 3 months on more than 3 days a week as a major complaint) were assessed via a phone interview with a research assistant. A total of 364 interested individuals were screened, and those who met the basic criteria ( $n=188$, all Caucasian) were invited to a second screening to assess the fear-avoidance and disability criteria. After being identified as eligible for the study, the participants were given an informed consent document that was discussed with the research assistant and could be taken home if participants needed more time to consider participation. The participants were not blinded to condition, and all three conditions were described in the consent document. Participants were informed of the possibility of adverse events, such as worsening of pain due to increased activity. We excluded 45 participants who did not meet inclusion criteria after the second screening. Thirty-three participants (some who met criteria and some who did not complete the screening) declined to participate prior to randomization. Eighty-eight participants were randomized to the three conditions (see Figure 1). As described in the "power analysis" section of our study protocol (Riecke et al., 2013), we aimed to include 108 participants to allow for detection of small effects. We were unable to reach this sample size due to the high number of approached patients who declined to participate prior to randomization.

Specific inclusion criteria were: Pain related anxiety and fear of movement were assessed by Tampa Scale of Kinesiophobia (TSK; Rusu, Kreddig, Hallner, Hülsebusch, \& Hasenbring, 2014) $\geq 35$, which has been used as a criterion in other studies of Exposure treatment in CLBP (Leeuw et al., 2008; J. W. Vlaeyen et al., 2001; Woods \& Asmundson, 2008). Additionally, with the help of experts, we developed a Photo Series of Daily Activities (PHODA)Profile (Leeuw, Goossens, van Breukelen, Boersma, \& Vlaeyen, 2007; Trost, France, \& Thomas, 2009). To be included, prospective participants had to rate perceived harmfulness as greater than 50 (range $0-100$, with $0=$ not harmful at all and $100=$ extremely harmful for my back) for at least 13 activities shown in PHODA pictures, including 8 rated as greater than 80 in order to provide enough movements for Exposure treatment. A further inclusion criterion was a sufficient level of disability, as defined by Quebec Back Pain Disability Scale (QBPDS) $\geq 15$ (Kopec et al., 1996).
Exclusion criteria included: back surgeries during the last six months or planned surgeries, red flags (Underwood \& Buchbinder, 2013), inability to read or write in German, pregnancy, alcohol addiction, psychotic disorders, and another current psychological treatment. Individuals were also excluded if they were unable to attend sessions regularly for physical or psychological reasons. Medications were allowed, but participants were asked not to change regular medications until the 6-month follow-up (6MFU) and to refrain from medications taken "as needed" during the exposure phase of treatment.

\section{Design}

Patients were randomized to one of three conditions: longer version of Exposure treatment (Exposure-long; consisting of 15 sessions with 10 Exposure sessions), shorter version of Exposure treatment (Exposure-short, consisting of 10 sessions with 5 Exposure sessions), and standard CBT for pain (consisting of 15 sessions).

Randomization followed a predetermined and computergenerated schedule, prestratified by degree of pain catastrophizing (Pain Catastrophizing Scale, PCS; Meyer, Sprott, \& Mannion, 2008; Sullivan, Bishop, \& Pivik, 1995) and disability (Pain Disability Index, PDI; Pollard, 1984; Soer et al., 2013). The median scores on pain catastrophizing and disability from a previous study (Glombiewski, Hartwich-Tersek, \& Rief, 2010b) were used as cutoffs. Within each stratum, a randomized block design with a block size of 9 was used to ensure equal distribution of important patient characteristics. The randomization procedure was performed by a research assistant blind to condition who prepared sequentially numbered, sealed, opaque envelopes. As is common in psychological treatments, it was not feasible to blind patients or therapists to treatment condition. Participants were assessed four times: prior to treatment (pre), after 10 sessions (mid), after treatment (post), and approximately 6 months (6 MFU) after post. For Exposure-short, there were only three assessments, because the assessment point after Session 10 was a posttreatment assessment given that only 10 sessions were offered in this condition. Weekly treatment process assessments were also conducted, and results will be reported in a separate paper.

\section{Outcome Measures}

For outcomes related to pain, we followed the recommendations from the Initiative on Methods, Measurements, and Pain Assessment in Clinical trials (IMMPACT; Dworkin et al., 2005).

\section{Primary Outcome Measures}

There were two primary outcomes, pain disability and pain intensity. Pain disability was assessed by two different measures: the QBPDS (Kopec et al., 1996), and the PDI (Pollard, 1984). Pain intensity was assessed with the NRS.

The QBPDS measures functional disability in daily activities for patients with low back pain. The QBPDS measures daily limitations due to pain more explicitly than do other disability or functioning measures, such as the PDI, making the QBPDS an especially suitable outcome measure for active pain treatments such as Exposure. The scale consists of one question: "Do you 


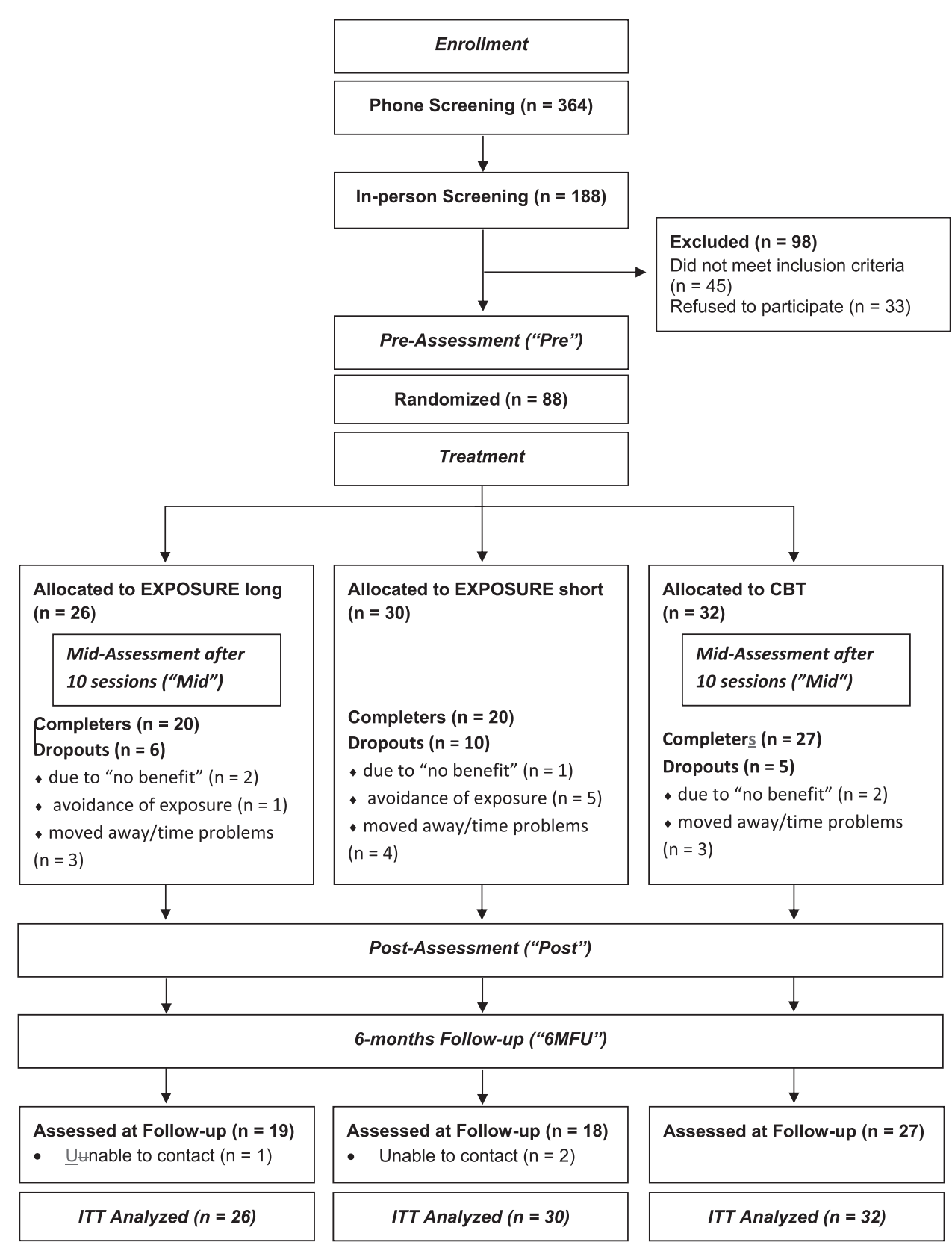

Figure 1. Flow of participants. $\mathrm{CBT}=$ cognitive behavioural therapy; Exposure $=$ Exposure for pain therapy; ITT $=$ Intention-to-Treat.

have trouble today with . . .?" followed by a list 20 activities of daily life (e.g., "taking something out of the fridge"). Those 20 activities are rated on a 5 -point scale $(0=$ no effort, $5=$ not able to). The summary score ranges from 0 to 100 , with higher scores indicating greater levels of functional disability. The QBPDS has strong psychometric properties. For this study, we translated the QBPDS into German and validated it using a larger sample (Riecke, Holzapfel, Rief, Lachnit, \& Glombiewski, 2016). The internal validity of the German version in this sample was high (Cronbach's alpha $=.94)$.

We also used the most popular instrument for measuring disability in pain, the PDI (Tait \& Chibnall, 2005), in order to allow for comparison of our results with results from other pain studies. The PDI is a 7-item self-report instrument assessing subjective degree of disability resulting from pain-related interference with daily activities. The PDI is less specific compared with the QBPDS: each of the 7 items asks for a general rating from $0=n o$ disability to $10=$ worst disability for 7 domains such as "social activities" or "occupation." The PDI has very good psychometric properties (Soer et al., 2013). Pain intensity is a mandatory outcome measure for all pain studies (Dworkin et al., 2005), although in psychological pain treatments we consider changes in pain intensity to be less relevant than changes in pain-related disability. Pain intensity was assessed using an 11-point scale from the 
German Pain Questionnaire (DSF) that assess average pain intensity during the past 4 weeks (Nagel, Gerbershagen, Lindena, \& Pfingsten, 2002).

\section{Secondary Outcome Measures}

Since this study is the first to compare CBT and Exposure for pain, we assessed several secondary and tertiary outcomes for exploratory analyses (see, Riecke et al., 2013, published protocol). In this paper we report on a small selection of the secondary measures that was based on the theories and treatment rationales underlying Exposure and CBT. We examined pain-related anxiety (Pain Anxiety Symptom Scale, PASS-20; McCracken \& Dhingra, 2002; Roelofs et al., 2004) and psychological flexibility/avoidance (Psychological Inflexibility in Pain Scale, PIPS; Wicksell, Lekander, Sorjonen, \& Olsson, 2010) since these constructs are targets of Exposure, but not CBT. We also examined coping strategies (Fragebogen zur Erfassung von Schmerzverarbeitung/ Bewältigung, FESV_BW; Geissner, 1999), as teaching coping strategies such as relaxation or attention shifting is the core of a CBT treatment, but is regarded as unnecessary or even counterproductive in an Exposure model. Finally, we examined depression (Hospital Anxiety and Depression Scale, HADS; Hinz \& Brahler, 2011), as this is the most frequent comorbidity in chronic pain, and it is important that pain treatments successfully target depression.

\section{Other Measures}

Demographic information was collected during the second screening.

Treatment expectancy and rationale credibility was measured using the Credibility and Expectancy Questionnaire (CEQ; Devilly \& Borkovec, 2000) at midtreatment. The therapeutic alliance was measured using 13 items on an 11-point scale $(0=$ strongly disagree, $10=$ absolutely agree). Treatment satisfaction was measured using 10 items on the same 11-point scale. Side effects were measured using the Inventory for the Assessment of Negative Effects of Psychotherapy (INEP; Ladwig, Rief, \& Nestoriuc, 2014) at $6 \mathrm{MFU}$.

A standardized dropout evaluation was developed. If a patient dropped out of treatment, a research assistant interviewed the participant about the reasons for discontinuing treatment and possible adverse effects of treatment. A follow-up assessment was conducted approximately six months after discontinuation.

All sessions were videotaped and evaluated for treatment fidelity by two trained master's students who were not involved in the study and who were blind to the hypotheses and to the treatment condition. The fidelity evaluation was based on Leeuw and colleagues' method of assessing treatment delivery in clinical trials (MATD; Leeuw, Goossens, de Vet, \& Vlaeyen, 2009). We adapted some MATD items according to our needs, but followed most of Leeuw and colleagues' recommendations. According to Leeuw and colleagues, each treatment element was classified into one of the following categories for each condition: (1) essential and unique; (2) essential but not unique; (3) compatible but not essential and not unique; and (4) prohibited. Essential and unique elements are only allowed in one treatment but not in the other; otherwise the treatment can be seen as "contaminated."
We chose three sessions to be examined for fidelity: Session 2 (general education about pain, equal for both conditions), Session 3 (Exposure: Education about the fear avoidance model, individualized fear-avoidance model, CBT: goal setting) and Session 6 (Exposure: Exposures/behavioral experiments; CBT: the second activity pacing session). All available videos from these sessions were watched by the two independent raters. The videos were stopped every $10 \mathrm{~min}$ in order to rate the occurrence of treatment elements. Once detected the element was rated as "did occur" for the whole session.

Agreement between raters was assessed by calculating Cohen's kappa.

\section{Treatments}

Participants in the Exposure-long and CBT groups received 15 weekly 50-min individual therapy sessions. Participants in the Exposure-short group received 10 weekly 50-min individual therapy sessions. The treatment was based on detailed manuals. The majority of participants completed treatment within 18-25 weeks. The treatment length did not differ between the groups and did not influence the results.

\section{Exposure Therapy}

Graded in vivo Exposure for pain aims to reduce pain-related disability by helping individuals to overcome fear of movements. This treatment is strongly approach-oriented; avoidance of movement, social activities, and other experiences is regarded as the major problem in chronic pain conditions. Coping strategies such as relaxation or attention shifting are not included in Exposure treatment for pain. In this study, we offered two versions of Exposure: a long form with 15 sessions (10 of which included Exposures) and a short form with 10 sessions (5 of which included Exposures).

The first four sessions included biographical assessment (1 session), psychoeducation about pain (1 session), psychoeducation about the fear-avoidance model and development of an individualized fear-avoidance model (1 session), and fear-hierarchy/goal setting (1 session). The fear hierarchy was generated using the Photo Series of Daily Activities (Trost et al., 2009) in which 100 pictures showing daily activities were rated for perceived harmfulness of the movement. In the subsequent 5 (Exposure-short) or 10 (Exposure-long) sessions, participants were encouraged to perform feared movements, and were encouraged to engage in these activities as much as possible between sessions until anxiety levels decreased. Behavioral experiments were integrated to challenge catastrophic beliefs about the consequences of pain or of specific movements. One session at the end of treatment was devoted to relapse prevention. The main mechanism of treatment is believed to be reduction in fear through learning that feared consequences such as injuries or unbearable pain are unlikely to occur.

\section{CBT}

The primary goal of the CBT approach is the same as that of Exposure treatment, that is, to decrease disability. However, different methods are used to achieve this goal. CBT encourages patients to develop an adaptive style of coping by maintaining a 
problem-solving orientation rather than emphasizing approach to feared stimuli. As in Turk's definition of CBT for pain (Turk, 2003), the treatment included three components: behavioral (2 sessions and then ongoing activity pacing), respondent (2 sessions of progressive muscle relaxation), and cognitive (3 sessions of cognitive restructuring and attention shift training). Additionally, treatment included a biographical assessment (1 session), psychoeducation about chronic pain and CBT models for chronic pain (1 session), goal setting (1 session), behavioral analysis (1 session), education about navigating the health care system (1 session), and a final relapse prevention session. Sessions 13 and 14 were flexible and could be adjusted to patients' needs (although providing Exposure treatment was forbidden).

\section{Therapists}

Study therapists were two advanced clinical psychology doctoral students who were in the middle of their training in CBT. They completed an additional intensive 2-day workshop on CBT for pain taught by Julia Anna Glombiewski and two 1-day workshops on Exposure for pain taught by Johan W. S. Vlaeyen and Jeroen de Jong, both highly experienced in delivering Exposure for pain. All treatments were supervised in weekly group supervision sessions by supervisors experienced in both CBT and Exposure for pain.

\section{Manuals}

We translated and adapted the protocol by Johan W. S. Vlaeyen and Jeroen de Jong (J. W. Vlaeyen et al., 2001; J. Vlaeyen, Morley, Linton, Boersma, \& de Jong, 2012) for implementing Exposure in a German outpatient setting. The CBT manual was developed by Julia Anna Glombiewski based on manuals used in another study on CBT in chronic pain (Glombiewski et al., 2010b) and those used in the Outpatient Center for Psychotherapy in the Department of Psychology at the University of Marburg, Germany.

\section{Statistical Method}

Missing data, including missing data as a result of drop-outs, were replaced when necessary by the multiple imputation procedure using the regression method under the assumption of data missing at random (MAR), as recommended in recent studies (Bhaskaran \& Smeeth, 2014).

We analyzed the data in three different ways. First, we computed effect sizes (Cohen's $d$ ) to establish the magnitude of the pre-to-post effects. Second, we computed reliable and clinically significant change as recommended by Jacobson and Truax (Jacobson \& Truax, 1991). Reliable change indices were calculated using the Reliable Change Index (RCI; Christensen \& Mendoza, 1986). The methods outlined by Jacobson and Truax (1991) were used to calculate cut-off scores for clinical significance. We defined two possible criteria for clinically significant change: (A) the participant's score moved 2 standard deviations away from the dysfunctional population mean; or (B) the post treatment scores placed the participant closer to the functional population mean than the dysfunctional population mean. We performed $\mathrm{Chi}^{2}$ analyses to compare the rates of clinically improved participants across the CBT and Exposure conditions.
Third, we analyzed the data using two longitudinal multilevel models for each outcome measure, with time of measurement (level 1) nested within patients (level 2) and therapy group as a level-2 predictor. With the first model, we tested whether participants in the three different therapy conditions showed different rate of change between baseline ("Pre") and the second point of measurement ("Mid"), to investigate whether Exposure-short led to faster symptom reduction (Hypothesis 2) as described by Barkham and colleagues (Barkham et al., 2006). In that model, the level-1 predictor time (one dummy variable), as well as the level-2 predictor therapy group (two dummy variables) were recoded as dichotomous dummy variables with pretreatment and Exposureshort as the respective reference categories. Most interestingly, the fixed slopes of the interaction terms indicate whether the estimated change between pre- and midtreatment differs between Exposurelong and Exposure-short (Dummy time $\times$ Dummy Group 1) as well as between CBT and Exposure-short (Dummy time $\times$ Dummy Group 2).

The second model aimed to test whether change between pre, post, and 6MFU depended on the type of therapy (Hypothesis 1). As we did not expect a linear trend over time, time was coded with two dummy variables: dummy Time 1 (which contrasts pre and post) and dummy Time 2 (which contrasts pre and 6MFU). We used a different coding scheme for type of therapy than was used in the first model, as we sought to compare the Exposure therapies individually against CBT. Hence, CBT was treated as the reference category and was contrasted either with Exposure-short (dummy Group 1) or Exposure-long (dummy Group 2). The fixed slopes of dummy Group 1 and dummy Group 2 indicate the estimated differences between Exposure-short and CBT at pretreatment (dummy Group 1) and between Exposure-long and CBT at Pretreatment (dummy Group 2). The fixed slopes of dummy Time $1 \times$ Dummy Group 1 and Dummy Time $1 \times$ Dummy Group 2 indicate whether change between pre and post differs between CBT and Exposure-short as well as between CBT and Exposure-long, respectively. The same applies to the contrast between pre and $6 \mathrm{MFU}$ for the fixed slopes of the interactions. Each dependent variable was tested individually using both models. In the random part of the models, all variances and covariances were freely estimated. The fixed intercept gives the estimated value. (See the online supplemental materials that contain the fixed parameters for each model.)

\section{Results}

\section{Demographic Information}

Participants' demographic characteristics are displayed in Table 1. The sample is comparable with other pain study samples and treatment-seeking pain populations.

\section{Treatment Expectancy and Credibility, Treatment Satisfaction, and Therapeutic Alliance}

As noted above, there was no true mid-treatment assessment for the Exposure-short condition, and thus we compared only Exposure-long to CBT at mid-treatment (see Figure 1). A metaanalysis of variance (MANOVA) with the CEQ credibility and expectancy scales, treatment satisfaction, and therapeutic alliance 
Table 1

Demographic Characteristics

\begin{tabular}{lccc}
\hline \multicolumn{1}{c}{ Variables* } & $\begin{array}{c}\text { CBT }(n=27 \\
\text { completers }+5 \\
\text { drop-outs })\end{array}$ & $\begin{array}{c}\text { Exposure-short } \\
(n=20+6)\end{array}$ & $\begin{array}{c}\text { Exposure-long } \\
(n=20+10)\end{array}$ \\
\hline Age & $53.5 \pm 9.0$ & $51.8 \pm 9.2$ & $52.7 \pm 9.4$ \\
Gender (\% female) & $71.9 \%$ & $50.0 \%$ & $38.5 \%$ \\
Education & $21.9 \%$ & $36.7 \%$ & $30.8 \%$ \\
$\quad$ Low & $43.8 \%$ & $30.0 \%$ & $30.8 \%$ \\
Middle & $34.4 \%$ & $33.3 \%$ & $38.5 \%$ \\
$\quad$ High & $56.3 \%$ & $40.0 \%$ & $46.2 \%$ \\
Work status & $9.4 \%$ & $6.7 \%$ & $23.1 \%$ \\
$\quad$ Self-)employed & $6.3 \%$ & $10.0 \%$ & $3.8 \%$ \\
$\quad$ Unemployed & $6.3 \%$ & $16.7 \%$ & $19.2 \%$ \\
Stay-at-home & $18.8 \%$ & $46.7 \%$ & $30.7 \%$ \\
Retirement pension & $25.0 \%$ & $12.94 \pm 10.1$ & $16.0 \pm 11.2$ \\
$\quad$ Disability pension & $15.1 \pm 11.5$ & $6.1 \pm 1.7$ & $5.8 \pm 2.1$ \\
Previous back surgeries & $5.6 \pm 1.6$ & $46.8 \pm 15.2$ & $46.8 \pm 17.3$ \\
Duration of pain (in years) & $44.3 \pm 15.1$ & $35.9 \pm 11.7$ & $32.3 \pm 11.3$ \\
Pain intensity & $33.9 \pm 12.4$ & & \\
QBPDS & $7.5 \pm 3.8$ & $10.1 \pm 4.6$ & $8.8 \pm 4.5$ \\
PDI & $9.3 \pm 4.2$ & $10.0 \pm 3.6$ & $9.8 \pm 4.5$ \\
HADS & & & \\
$\quad$ Depression & Anxiety & &
\end{tabular}

Note. Values are presented as means $( \pm$ standard deviation) or percentages. There were significantly fewer women in both Exposure-short, $\chi^{2}(1)=7.1, p=.008$ and Exposure-long, $\chi^{2}(1)=14.36, p<.001$ than in CBT. Exposure-long and Exposure-short did not differ on sex distribution, $\chi^{2}(1)=1.39, p=.30$. CBT = cognitivebehavioral therapy; QBPDS = Quebec Back Pain Disability Scale; PDI = Pain Disability Index; HADS = Hospital Anxiety and Depression Scale.

* Completers and dropouts did not differ on any of the variables shown in the table other than gender.

as dependent variables and group (CBT vs. Exposure-long) as a factor revealed a significant effect for group, $F(4,53)=3.87, p=$ .008. Post hoc analyses indicated that Exposure treatment was perceived as less credible (CEQ credibility scale) than CBT at mid-treatment, $F(1,56)=7.12, p=.01$. At post-treatment, the two groups did not differ on CEQ, treatment satisfaction, or treatment alliance scores, $F(8,166)=.72, p=.67$.

\section{Side Effects}

All documented side effects are presented in the online supplemental materials. Seventeen participants (9 in Exposure, 8 in CBT) experienced some side effects. In the Exposure conditions, the side effects that participants explicitly attributed to treatment were: being more troubled by the past $(n=1)$, worsened relations with family $(n=1)$, being anxious that colleagues or friends might find out about the therapy $(n=2)$, having troubles finding insurance $(n=1)$, having more financial worries $(n=3)$, feeling addicted to the therapist $(n=1)$, having problems making important decisions without the therapist $(n=1)$, experiencing "downs" during treatment $(n=3)$, suffering from suicidal thoughts $(n=1)$, and feeling that the therapist forced the patient to do things that the patient did not want him or her to do $(n=2)$. In CBT, the participants experienced following the side effect of treatment: being more troubled by the past $(n=2)$, being anxious that colleagues or friends might find out about the therapy $(n=2)$, having more financial worries $(n=1)$, experiencing "downs" during treatment $(n=5)$, feeling addicted to the therapist $(n=2)$, having problems making important decisions without the therapist $(n=1)$, and feeling hurt by statements made by the therapist $(n=1)$. The total number of side effects and total number of individuals experiencing side effects did not differ across Exposure and CBT. No patient reported any injuries due to Exposures.

\section{Dropout Evaluation}

Twenty-one participants (14 women and seven men, 16 in Exposure-long/-short and 5 in CBT) dropped out of treatment. A $\mathrm{Chi}^{2}$ test revealed that there were significantly more dropouts in the Exposure treatments (combined for this analysis) than in the CBT treatment, $\mathrm{Chi}^{2}(1)=7.12, p=.008$. There were proportionally more women among dropouts than among completers, and this difference was statistically significant, $\mathrm{Chi}^{2}(1)=7.64, p=.006$. The dropouts did not differ from completers on demographic variables and baseline pain-related questionnaire scores, as shown in Table 1. Dropouts reported lower treatment alliance than completers at the pre-treatment assessment, $F(1 ; 87)=11.66, p=$ .001 . We were able to interview all 21 dropouts about their reasons for discontinuing treatment (see Figure 1). Three of these participants in the Exposure conditions and two in the CBT condition reported that they did not find the treatment beneficial. Six participants reported dropping out because they wanted to avoid Exposures. Ten of the 21 participants completed a follow-up questionnaire 6 months after dropping out. Exploratory $t$ test analyses revealed that they experienced reduced disability (measured by the QBPDS) compared with pretreatment (pre: $M=53.6$, $S D=14.05$; post: $M=44.5, S D=15.31, t(9)=2.86, p=.019)$. Only one of the 10 dropouts reported side effects due to treatment at follow-up. 


\section{Treatment Fidelity}

According to Leeuw and colleagues (Leeuw et al., 2009), protocol adherence is defined as the occurrence of at least $70 \%$ of essential treatment elements. Treatment contamination is defined as the occurrence of at least $10 \%$ of prohibited treatment elements. Treatment differentiation (i.e., the presence of sufficient differences between the two treatments) was considered to be achieved if more than $90 \%$ of sessions were correctly classified (as Exposure or CBT). Session two was excluded from the differentiation rating because it was identical for $\mathrm{CBT}$ and Exposure. For some sessions, videos were missing due to technical or logistical problems, for example, when Exposures took place outside the treatment building. Videos were available for 167 sessions, including both completers and dropouts (64 videos of Session 2, 61 of Session 3, and 42 of Session 6). Sixteen of those videos could not be rated due to poor quality or unclear session number. Thus, the final video sample included 151 videos of 78 study participants.

Cohen's kappa for all ratings was moderate $(0.57(p<.01$; Landis \& Koch, 1977). The mean protocol adherence scores are reflected in the mean proportion of essential treatment elements over all rated treatment sessions (see the online supplemental materials). General treatment adherence over the three session was $69.7 \%$ for CBT and $66.5 \%$ for Exposure, minimally undercutting the $70 \%$ criterion recommended by Leeuw and colleagues (Leeuw et al., 2009). Treatment adherence did not differ between treatments, $t(49)=0.9 ; p=.37$. Treatment contamination in sessions three and six occurred in $21 \%$ of cases. Treatment differentiation was high:
Session 3 was correctly identified as CBT in $96 \%$ of cases and as Exposure in $92 \%$ of cases. Session 6 was correctly identified as CBT in $94 \%$ of cases and as Exposure in $100 \%$ of cases.

\section{Pretreatment Group Differences}

No significant pretreatment differences were found between CBT and Exposure on any primary or secondary outcome measures.

\section{Effect Sizes}

Table 2 displays pre-post and pre-6MFU effect sizes (Cohen's d) and $95 \%$ confidence intervals. All effect sizes were significant, indicating improvements from pre to post and from pre to 6MFU. Disability was reduced with large effect sizes in Exposure-short and Exposure-long and with moderate to large effect sizes in CBT.

\section{Reliable and Clinically Significant Change}

For analyses of reliable and clinically significant change for the QBPDS, we used the test-retest reliability of .92 and the pretreatment mean $(M=45.6, S D=15.66)$ from the Kopec et al. study (Kopec et al., 1996). No normative data from the general population are available for the QBPDS. The reliable change criterion was determined as a change of greater than or equal to 12.28 , and the threshold for clinically significant change (criterion purely in terms of clinical distribution) was 14.28; accordingly, we selected change of 14.20 or more as the criterion for reliable and clinically

Table 2

Effect Sizes (Cohen's D) From Pre-to Post-Treatment and Pre-Treatment to 6-Months Follow-Up for All Relevant Outcome Variables

\begin{tabular}{|c|c|c|c|c|c|c|c|c|}
\hline \multirow[b]{2}{*}{ Outcome } & \multicolumn{2}{|c|}{ Pre } & \multicolumn{2}{|c|}{ Post } & \multicolumn{2}{|c|}{$6 \mathrm{MFU}$} & \multicolumn{2}{|c|}{ ES $[95 \% \mathrm{CI}]$} \\
\hline & $M$ & $S D$ & $M$ & $S D$ & $M$ & $S D$ & Pre-Post & Pre-6MFU \\
\hline \multicolumn{9}{|l|}{ CBT } \\
\hline QBPDS & 43.70 & 15.22 & 34.26 & 18.51 & 29.54 & 17.49 & $.54[.04,1.04]$ & $.87[.36,1.38]$ \\
\hline PDI & 32.99 & 12.07 & 20.41 & 12.95 & 18.23 & 11.26 & $\mathbf{1 . 0 0}[.48,1.52]$ & $\mathbf{1 . 2 2}[.68,1.75]$ \\
\hline Pain intensity & 5.56 & 1.65 & 4.60 & 2.29 & 3.91 & 2.32 & $.63[.13,1.13]$ & $\mathbf{1 . 2 6}[.73,1.80]$ \\
\hline PASS-20 & 40.72 & 17.82 & 30.33 & 16.69 & 26.79 & 14.79 & $\mathbf{. 6 1}[.10,1.12]$ & $\mathbf{. 9 0}[.38,1.41]$ \\
\hline PIPS & 51.54 & 11.94 & 45.73 & 12.33 & 43.77 & 13.68 & $.52[.02,1.02]$ & $.66[.16,1.17]$ \\
\hline FESV_BW & 79.74 & 13.51 & 97.40 & 20.29 & 98.03 & 22.32 & $\mathbf{1 . 0 7}[.50,1.60]$ & $\mathbf{1 . 0 5}[.53,1.57]$ \\
\hline HADS depression & 7.47 & 3.78 & 5.96 & 4.15 & 5.54 & 3.67 & $.57[.07,1.07]$ & $.67[.16,1.17]$ \\
\hline \multicolumn{9}{|l|}{ Exposure-short } \\
\hline QBPDS & 46.83 & 15.24 & 30.42 & 13.15 & 28.00 & 12.94 & $\mathbf{1 . 1 4}[.59,1.69]$ & $\mathbf{1 . 3 3}[.77,1.88]$ \\
\hline PDI & 36.03 & 11.31 & 19.83 & 8.53 & 20.33 & 9.25 & $\mathbf{1 . 7 7}[1.17,2.36]$ & $\mathbf{1 . 5 9}[1.01,2.17]$ \\
\hline Pain intensity & 6.07 & 1.68 & 4.54 & 1.33 & 4.21 & 1.98 & $\mathbf{1 . 0 1}[.47,1.55]$ & $\mathbf{1 . 1 3}[.58,1.67]$ \\
\hline PASS-20 & 43.07 & 17.96 & 27.28 & 13.06 & 31.00 & 13.35 & $\mathbf{1 . 0 6}[.52,1.60]$ & $.79[.27,1.32]$ \\
\hline PIPS & 58.45 & 12.35 & 42.69 & 9.37 & 44.15 & 9.60 & $\mathbf{1 . 5 1}[.94,2.08]$ & $\mathbf{1 . 3 2}[.76,1.88]$ \\
\hline FESV_BW & 73.74 & 17.10 & 82.31 & 10.65 & 83.1 & 10.17 & $.65[.13,1.16]$ & $.72[.20,1.24]$ \\
\hline HADS depression & 10.10 & 4.61 & 8.10 & 3.98 & 7.73 & 3.82 & $.57[.05,1.08]$ & $.85[.32,1.38]$ \\
\hline \multicolumn{9}{|l|}{ Exposure-long } \\
\hline QBPDS & 46.51 & 17.00 & 27.55 & 18.13 & 25.69 & 14.76 & $\mathbf{1 . 0 9}[.50,1.67]$ & $\mathbf{1 . 3 5}[.75,1.95]$ \\
\hline PDI & 31.84 & 11.31 & 20.01 & 11.51 & 18.97 & 11.98 & $\mathbf{1 . 0 0}[.42,1.58]$ & $\mathbf{1 . 1 8}[.59,1.77]$ \\
\hline Pain intensity & 5.81 & 2.02 & 4.79 & 1.89 & 4.08 & 2.38 & $.63[.08,1.20]$ & $.78[.22,1.35]$ \\
\hline PASS-20 & 45.27 & 19.53 & 33.37 & 19.97 & 29.37 & 17.35 & $\mathbf{1 . 5 3}[.91,2.14]$ & $\mathbf{1 . 3 9}[.78,1.99]$ \\
\hline PIPS & 56.35 & 12.13 & 44.55 & 13.56 & 42.39 & 14.15 & $\mathbf{. 9 6}[.39,1.53]$ & $\mathbf{1 . 0 7}[.49,1.66]$ \\
\hline FESV_BW & 80.99 & 20.00 & 91.91 & 16.09 & 87.98 & 17.47 & $.62[.07,1.18]$ & $.38[.16, .94]$ \\
\hline HADS depression & 8.81 & 4.49 & 5.96 & 4.46 & 6.52 & 4.05 & $.75[.19,1.31]$ & $\mathbf{. 5 0}[-.05,1.05]$ \\
\hline
\end{tabular}

Note. $\quad$ CBT $=$ cognitive-behavioral therapy; QBPDS = Quebec Back Pain Disability Scale; PDI = Pain Disability Index; PASS-20 = Pain Anxiety Symptom Scale; PIPS = Psychological Inflexibility in Pain Scale; FESV_BW = Fragebogen zur Erfassung von Schmerzverarbeitung/Bewältigung; HADS $=$ Hospital Anxiety and Depression Scale. 
significant change. For the PDI, we used the test-retest reliability (intraclass correlation coefficient of 0.91) from the Grönblad et al. (Grönblad et al., 1993) study, pretreatment mean $(M=33.69$, $S D=11.59)$ and normative population data $(M=6.8, S D=11.4)$ from the Mewes et al. study (Mewes et al., 2009). The reliable change criterion was determined to be a change of 9.64 or more, and the threshold for clinically significant change was 10.51 (criterion purely in terms of clinical distribution, criterion "A"). The reliable change criterion based on both the clinical distribution and normative data (criterion "C") was a change of 20.13 or more. For the numeric Pain Intensity scale, a change of at least 1.5 points (or approximately 20\%) is commonly regarded as being clinically significant (Kovacs et al., 2007).

For the primary outcome measure of disability (measured using the QBPDS), Exposure-short and Exposure-long both led to significantly higher rates of reliable and clinically significant change compared to CBT at both posttreatment and 6MFU (see Table 3).

\section{Differential Efficacy of CBT and Exposure Treatments (Hypothesis 1)}

Linear mixed models revealed significant improvement on all outcome measures from pretreatment to posttreatment and from pretreatment to $6 \mathrm{MFU}$ (see online supplemental materials). We expected Exposure to be more effective than CBT at reducing disability, and this hypothesis was partially supported. For the QBPDS, Exposure-long was more effective than CBT at reducing disability from pre- to posttreatment (Dummy Time $1 \times$ Dummy Group 2: $b=-9.52, S E=4.05, t=-2.35, p=.05)$, in line with the results of the clinical significance analysis. The difference between Exposure-long and CBT at follow-up and differences between Exposure-short and CBT trended toward significance. However, there were no differences between CBT and the Exposure treatments for other primary outcome measures.

Analyses of other measures indicated that Exposure-short was more effective than CBT at enhancing psychological flexibility in the short- and long-term (Dummy Time $1 \times$ Dummy Group 1: $b=-9.95, S E=3.03, t=-3.29, p<.001$; Dummy Time $2 \times$ Dummy Group 1: $b=-6.53, S E=3.15, t=2.07, p=.04)$. Results for Exposure-long trended toward significance ( $p$ values $=$ .06). CBT was more effective than Exposure-short at increasing pain-related coping strategies at posttreatment (Dummy Time $1 \times$ Dummy Group 1: $b=-9.09, S E=4.28, t=-2.12, p=.04)$ and at 6MFU (Dummy Time $2 \times$ Dummy Group 1: $b=-8.92, S E=$ $4.46, t=-2, p=.05)$. CBT was also more effective than Exposure-long at increasing pain-related coping at posttreatment (Dummy Time $2 \times$ Dummy Group 2: $b=-11.31, S E=4.63$, $t=-2.44, p=.2)$.

\section{Comparison of Treatments After 10 Sessions (Hypothesis 2)}

After 10 sessions, participants in CBT and Exposure-long were in the middle of the active treatment, while participants in Exposure-short had completed treatment. The comparison of Exposure-short with Exposure-long at this time point indicated significantly greater improvements in the Exposure-short condition for disability (QBPDS: Dummy time $\times$ Dummy Group 1: $b=$ 8.01, $S E=3.8, t=2.11, p=.04$; PDI: Dummy time $\times$ Dummy

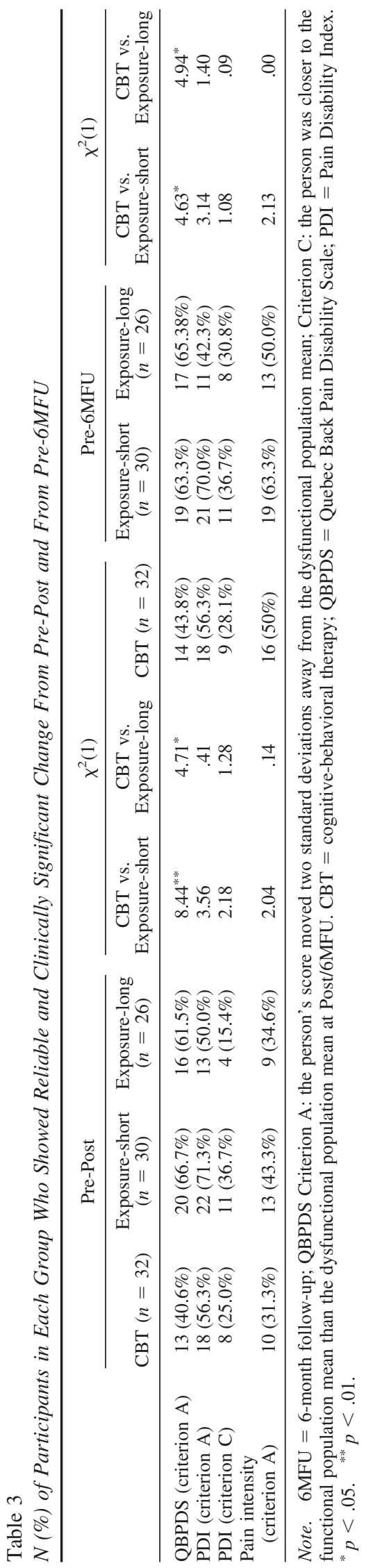


Group 1: $b=6.1, S E=2.96, p=.04$ ), pain intensity (Dummy group time $\times$ Dummy Group 1: $b=1.1, S E=.46, t=2.42, p=$ .02 ), pain-related fear (Dummy time $\times$ Dummy Group 1: $b=$ 8.26, $S E=4, t=2.07, p=.04)$, and psychological flexibility (Dummy time $\times$ Dummy Group 1: $\mathrm{b}=11.68, S E=2.73, t=$ $4.28, p<.001)$. These results support the hypothesis that individuals recover faster when they are offered fewer sessions.

The comparison of Exposure-short with CBT after 10 sessions indicated that Exposure-short led to significantly greater improvements in disability (QBPDS: Dummy time $\times$ Dummy Group 2: $b=7.8, S E=3.13, t=2.5, p=.01$ ), pain related fear (Dummy time $\times$ Dummy Group 2: $b=10.38, S E=4.22, t=2.46, p=$ .02 ), and psychological flexibility (Dummy time $\times$ Dummy Group $2: b=7.25, S E=2.88, t=2.52, p=.01)$.

\section{Discussion}

This study is the first to compare a traditional psychological approach for CLBP with a less common approach (Exposure) tailored for individuals who avoid movements due to fear of harming their backs. Exposure was effective with large pre-post and pre-follow-up effect sizes in all outcome domains (e.g., pain intensity, disability). Exposure was more effective than CBT at reducing movement-related disability based on a clinical significance assessment, and these results were partly confirmed by a linear mixed models analysis. Over $60 \%$ of participants in the Exposure conditions achieved clinically significant reduction in disability (assessed with the QBPDS) at follow-up, compared with $44 \%$ in the CBT condition. In addition, Exposure led to greater improvements in psychological flexibility than did CBT; this is in line with the idea that the core feature of psychological flexibility is "confrontation instead of avoidance" (Wicksell et al., 2010), consistent with the theory of Exposure. The most important result, in our opinion, was that Exposure was effective after only 10 sessions (of which 5 were Exposures). However, Exposure was also experienced as less credible than CBT during treatment, and had a significantly higher dropout rate (28\%) compared with CBT (16\%). Approximately 30\% of participants reported side effects, and these participants were equally distributed across CBT and Exposure treatments.

CBT was also effective, with medium to large pre-post and pre-6MFU effect sizes. Analyses of the PDI, one of two measures used to assess disability, suggested that CBT was as effective as Exposure in reducing pain-related disability. In addition, CBT was more effective than Exposure at enhancing pain-related coping strategies, consistent with the theoretical rationale of CBT.

Thus, our results suggest that in order to best tailor treatment offerings, Exposure should be preferentially offered to individuals suffering from high levels of disability and fear of movement. Individuals who are relatively weak on coping strategies might specifically benefit from CBT. However, we cannot determine whether individuals with low fear of movement might also benefit from Exposure, since we only included highly fearful-avoidant participants. Further research should address this question.

Treatment adherence by therapists was at approximately $70 \%$ in both treatments, which is considered satisfactory. The treatment adherence ratings left room for improvement, as some sessions were "contaminated" by the presence of elements from the other treatment (for instance, the presence of cognitive restructuring in the Exposure conditions). Nevertheless, the results of treatment fidelity ratings suggest that two distinct treatments were conducted.

Overall, both treatments appeared to be equally effective: the slight advantages of Exposure on one primary outcome measure of disability were counterbalanced by the lower dropout rate and better treatment credibility ratings in CBT. Also, Exposure only outperformed CBT in reducing disability on one of the two disability measures, the QBPDS. We believe that QBPDS is better suited than the PDI to measure everyday functional limitations, as the items very specifically assess the daily problems of individuals with low back pain. Nevertheless, the PDI and pain intensity rating do not show any advantages of Exposure over CBT-although the sample was tailored to Exposure-treatment

The pre-post and pre-follow-up effect sizes (see Table 2) are untypically large for psychological treatments in chronic pain. Usually, the effects of CBT for CLBP are moderate, around $d=.6$ (Chou et al., 2017; Morley et al., 1999). We suggest two explanations for this phenomenon. First, we thoroughly followed recommendations provided by colleagues such as Eccleston, Williams, and Morley (Williams, Eccleston, \& Morley, 2012), and placed the highest emphasis on the selection, training, and supervision of our two study therapists. All supervisors and trainers were highly experienced in both conducting RCTs for pain and teaching and delivering Exposure and CBT for chronic pain. Supervision took place almost weekly, and session videos were watched and discussed in each supervision meeting. Although each therapists delivered both treatments, and often the same supervisor was responsible for supervising both Exposure and CBT, we believe there was a low probability of allegiance effects: all staff directly involved in the study in Marburg were trained and skilled in CBT but also had large expectations for Exposure, while the supervisors from Leuven and Maastricht were very experienced in Exposure and were convinced of its likely efficacy. Second, our media advertisements for the study emphasized "psychological treatment and being active," and thus participants may have begun the study with high motivation for change and openness to psychological approaches. This may weaken the generalizability of our findings to populations that are often involuntarily confronted with psychological treatments during their multidisciplinary treatment or rehabilitation, and therefore may not respond as well as our self-selecting study population.

Treatment delivery and supervision in this study was performed by psychologists and psychology trainees, indicating that Exposure can be safely and effectively delivered in a psychology clinic setting. However, while we did not collect quantitative data on this topic, our qualitative observations suggest that fear-avoidance beliefs on the part of psychologists resulted in challenges for the implementation of this treatment. In particular, psychologists may be susceptible to patients' portrayals of fragile backs, and therefore may be anxious themselves during first Exposures. Such fears might present a barrier for the dissemination of Exposure treatments for CLBP, as physical therapists and MDs are also susceptible to fear-avoidance beliefs (Linton, Vlaeyen, \& Ostelo, 2002).

At midtreatment, Exposure-short was more effective than CBT and Exposure-long in all core outcome domains. It is not surprising that CBT was less effective than Exposure at this time point, as core $\mathrm{CBT}$ interventions such as cognitive restructuring had not yet taken place. More intriguingly, Exposure-short outperformed 
Exposure-long after 5 sessions of Exposure, replicating the findings of Barkham and colleagues (Barkham et al., 2006) that individuals recover faster when they are offered fewer sessions. After the end of Exposure-long, the difference between the two Exposure treatments disappeared. These findings have two important clinical implications. First, when both therapists and patients know that the treatment will be short, they achieve better results faster than when they have more time available. This suggests that for Exposure treatments of chronic pain, brief treatments should be the standard, with a possibility of extension only with special justification. Second, Exposure treatments fit well within multidisciplinary (inpatient) treatments for chronic pain, for example, rehabilitation or multimodal pain treatments within the German health care system. Psychologists have limited time (e.g., 5 group sessions and 5 individual sessions) within these treatments to work with the patient, and a time-efficient Exposure treatment might be a good fit under these circumstances. However, this would require specialized assessment at pretreatment, since only patients with elevated levels of fear-avoidance have been shown to benefit from Exposure in our study. Also, as mentioned above, psychologists' potential fear-avoidance beliefs must be addressed in order to allow provision of effective treatment.

This study is the first to systematically investigate adverse events during psychological treatment of pain using a validated questionnaire at follow-up. Psychological treatments for pain are expected to produce fewer side effects then medications, which was supported in this study given that up to $80 \%$ of patients report side effects due to opioids (Sehgal, Colson, \& Smith, 2013). However, with approximately $30 \%$ of our participants reporting side effects, our results are similar to those of antidepressant studies (Cascade, Kalali, \& Kennedy, 2009), in which up to $40 \%$ participants report side effects. We encourage clinicians working with individuals with chronic pain to be attentive to potential side effects we found in this study. Specifically, eight participants felt that treatment significantly worsened their mood while it was delivered, while three participants felt addicted to the therapist and reported difficulties making decisions without the therapist six months after treatment. In the Exposure condition, two participants felt that they were forced to do things that they did not want to do. We recommend that clinicians inform patients in advance that side effects might occur, and to address any occurring problems as frank as possible.

In our study we deliberately did not include a treatment-as-usual or wait-list control group, as a number of studies have already compared treatments such as CBT and Exposure with a wait-list control group. However, we placed great importance on the dropout analysis, which indicated that several patients decided to avoid Exposures and that dropouts reported lower treatment alliance even at pre-treatment. This suggests that early administration of alliance measures might aid identification of potential dropouts. The dropout analysis also indicated that dropouts improved without continued treatment, suggesting that there may be a natural course of improvement that could raise questions about the large pre- to follow-up effect sizes that we reported. Another possible explanation is that participants who have a greater ability to improve without professional help are more likely to drop out of treatment (Glombiewski, Hartwich-Tersek, \& Rief, 2010a).

Strengths of this study include a thorough treatment fidelity assessment, side effects assessment, and dropout-analysis, the novel comparison of CBT and Exposure for pain as well as the comparison of different treatment lengths, and use of three different methods of analyzing efficacy (effect sizes, clinical significance, and linear mixed models analysis). The major limitation is the sample size: although the study sample is larger than those of other studies on Exposure, it is still smaller than has been suggested in recent calls for improved RCTs on CLBP (Eccleston, Morley, \& Williams, 2013). We also were not able to reach the $N$ that our power analysis suggested was needed to detect smaller differences among the three treatment arms. Although we were still able to demonstrate differences among treatments, there were several interesting trend-level results that were in line with our hypotheses and major findings, suggesting a lack of statistical power.

In summary, we recommend considering Exposure as a brief and effective treatment for reducing disability in individuals suffering from fear of movement and CLBP. Practitioners should expect higher attrition rates in Exposure and should monitor their own fear-avoidance beliefs. Our results also support the efficacy of CBT, including cognitive restructuring, activity pacing, and relaxation, for CLBP. We recommend careful assessment of side effects of CBT and Exposure for CLBP.

Future research should focus on multicenter studies in order to achieve larger samples. Potential directions for future research include identifying predictors of responsiveness to Exposure, extending Exposure-based treatments to other settings and populations (group treatment, treatment of children), addressing fearavoidance beliefs among practitioners involved in pain treatment, and developing and disseminating more effective CBT protocols for inpatient and outpatient settings. To conclude, our study demonstrates that Exposure for pain, although challenging for patients is a highly effective and economical therapy that can be delivered in a psychological treatment setting and should be offered as a short-term treatment.

\section{References}

Barkham, M., Connell, J., Stiles, W. B., Miles, J. N. V., Margison, F., Evans, C., \& Mellor-Clark, J. (2006). Dose-effect relations and responsive regulation of treatment duration: The good enough level. Journal of Consulting and Clinical Psychology, 74, 160-167. http://dx.doi.org/10 .1037/0022-006X.74.1.160

Bhaskaran, K., \& Smeeth, L. (2014). What is the difference between missing completely at random and missing at random? International Journal of Epidemiology, 43, 1336-1339. http://dx.doi.org/10.1093/ije/ dyu080

Boersma, K., Linton, S., Overmeer, T., Jansson, M., Vlaeyen, J., \& de Jong, J. (2004). Lowering fear-avoidance and enhancing function through exposure in vivo. A multiple baseline study across six patients with back pain. Pain, 108, 8-16. http://dx.doi.org/10.1016/j.pain.2003 .03 .001

Breivik, H., Collett, B., Ventafridda, V., Cohen, R., \& Gallacher, D. (2006). Survey of chronic pain in Europe: Prevalence, impact on daily life, and treatment. European Journal of Pain, 10, 287-333. http://dx .doi.org/10.1016/j.ejpain.2005.06.009

Cascade, E., Kalali, A. H., \& Kennedy, S. H. (2009). Real-world data on SSRI antidepressant side effects. Psychiatry, 6, 16-18.

Chou, R., Deyo, R., Friedly, J., Skelly, A., Hashimoto, R., Weimer, M., . . Brodt, E. D. (2017). Nonpharmacologic therapies for low back pain: A systematic review for an American College of Physicians Clinical Prac- 
tice Guideline. Annals of Internal Medicine, 166, 493. http://dx.doi.org/ $10.7326 / \mathrm{m} 16-2459$

Christensen, L., \& Mendoza, J. L. (1986). A method of assessing change in a single subject-An alteration of the RC Index. Behavior Therapy, 17, 305-308. http://dx.doi.org/10.1016/S0005-7894(86)80060-0

den Hollander, M., Goossens, M., de Jong, J., Ruijgrok, J., Oosterhof, J., Onghena, P., . . Vlaeyen, J. W. (2016). Expose or protect? A randomized controlled trial of exposure in vivo vs pain-contingent treatment as usual in patients with complex regional pain syndrome type 1. Pain, 157, 2318-2329. http://dx.doi.org/10.1097/j.pain.0000000000000651

Devilly, G. J., \& Borkovec, T. D. (2000). Psychometric properties of the credibility/expectancy questionnaire. Journal of Behavior Therapy and Experimental Psychiatry, 31, 73-86. http://dx.doi.org/10.1016/S00057916(00)00012-4

Dworkin, R. H., Turk, D. C., Farrar, J. T., Haythornthwaite, J. A., Jensen, M. P., Katz, N. P., . . W Witter, J. (2005). Core outcome measures for chronic pain clinical trials: IMMPACT recommendations. Pain, 113, 9-19. http://dx.doi.org/10.1016/j.pain.2004.09.012

Eccleston, C., Morley, S. J., \& Williams, A. C. (2013). Psychological approaches to chronic pain management: Evidence and challenges. British Journal of Anaesthesia, 111, 59-63. http://dx.doi.org/10.1093/bja/ aet207

Geissner, E. (1999). Coping with chronic pain - assessment of coping strategies and distress. Zeitschrift Fur Klinische Psychologie-Forschung Und Praxis, 28, 280-290. http://dx.doi.org/10.1026/0084-5345.28.4.280

Glombiewski, J. A., Hartwich-Tersek, J., \& Rief, W. (2010a). Attrition in cognitive-behavioral treatment of chronic back pain. The Clinical Journal of Pain, 26, 593-601. http://dx.doi.org/10.1097/AJP.0b013e3181e37611

Glombiewski, J. A., Hartwich-Tersek, J., \& Rief, W. (2010b). Two psychological interventions are effective in severely disabled, chronic back pain patients: A randomised controlled trial. International Journal of Behavioral Medicine, 17, 97-107. http://dx.doi.org/10.1007/s12529009-9070-4

Goossens, M. E., de Kinderen, R. J. A., Leeuw, M., de Jong, J. R., Ruijgrok, J., Evers, S. M., \& Vlaeyen, J. W. (2015). Is exposure in vivo cost-effective for chronic low back pain? A trial-based economic evaluation. BMC Health Services Research, 15, 549. http://dx.doi.org/10 .1186/s12913-015-1212-6

Grönblad, M., Hupli, M., Wennerstrand, P., Järvinen, E., Lukinmaa, A., Kouri, J. P., \& Karaharju, E. O. (1993). Intercorrelation and test-retest reliability of the Pain Disability Index (PDI) and the Oswestry Disability Questionnaire (ODQ) and their correlation with pain intensity in low back pain patients. The Clinical Journal of Pain, 9, 189-195. http://dx .doi.org/10.1097/00002508-199309000-00006

Hansen, N. B., Lambert, M. J., \& Forman, E. M. (2002). The psychotherapy dose-response effect and its implications for treatment delivery services. Clinical Psychology: Science and Practice, 9, 329-343. http:// dx.doi.org/10.1093/clipsy.9.3.329

Harnett, P., O'Donovan, A., \& Lambert, M. J. (2010). The dose response relationship in psychotherapy: Implications for social policy. Clinical Psychologist, 14, 39-44. http://dx.doi.org/10.1080/13284207.2010 .500309

Hasenbring, M. I., \& Verbunt, J. A. (2010). Fear-avoidance and endurancerelated responses to pain: New models of behavior and their consequences for clinical practice. The Clinical Journal of Pain, 26, 747-753. http://dx.doi.org/10.1097/AJP.0b013e3181e104f2

Hinz, A., \& Brahler, E. (2011). Normative values for the Hospital Anxiety and Depression Scale (HADS) in the general German population. Journal of Psychosomatic Research, 71, 74-78. http://dx.doi.org/10.1016/j .jpsychores.2011.01.005

Jacobson, N. S., \& Truax, P. (1991). Clinical significance: A statistical approach to defining meaningful change in psychotherapy research. Journal of Consulting and Clinical Psychology, 59, 12-19. http://dx.doi .org/10.1037/0022-006X.59.1.12
Kopec, J. A., Esdaile, J. M., Abrahamowicz, M., Abenhaim, L., WoodDauphinee, S., Lamping, D. L., \& Williams, J. I. (1996). The Quebec Back Pain Disability Scale: Conceptualization and development. Journal of Clinical Epidemiology, 49, 151-161. http://dx.doi.org/10.1016/ 0895-4356(96)00526-4

Kovacs, F. M., Abraira, V., Royuela, A., Corcoll, J., Alegre, L., Cano, A., ... Mufraggi, N. (2007). Minimal clinically important change for pain intensity and disability in patients with nonspecific low back pain. Spine, 32, 2915-2920. http://dx.doi.org/10.1097/BRS.0b013e31815b75ae

Ladwig, I., Rief, W., \& Nestoriuc, Y. (2014). Welche Risiken und Nebenwirkungen hat Psychotherapie-Entwicklung des Inventars zur Erfassung Negativer Effekte von Psychotherapie (INEP) [What are the risks and side effects to psychotherapy? - Development of an Inventory for the Assessment of Negative Effects of Psychotherapy (INEP)]. Verhaltenstherapie, 24, 252-263. http://dx.doi.org/10.1159/000367928

Landis, J. R., \& Koch, G. G. (1977). The measurement of observer agreement for categorical data. Biometrics, 33, 159-174. http://dx.doi org $/ 10.2307 / 2529310$

Leeuw, M., Goossens, M. E., de Vet, H. C. W., \& Vlaeyen, J. W. (2009). The fidelity of treatment delivery can be assessed in treatment outcome studies: A successful illustration from behavioral medicine. Journal of Clinical Epidemiology, 62, 81-90. http://dx.doi.org/10.1016/j.jclinepi 2008.03.008

Leeuw, M., Goossens, M. E., van Breukelen, G. J., Boersma, K., \& Vlaeyen, J. W. (2007). Measuring perceived harmfulness of physical activities in patients with chronic low back pain: The Photograph Series of Daily Activities-Short electronic version. The Journal of Pain, 8 840-849. http://dx.doi.org/10.1016/j.jpain.2007.05.013

Leeuw, M., Goossens, M. E., van Breukelen, G. J. P., de Jong, J. R., Heuts, P. H., Smeets, R. J., . . Vlaeyen, J. W. (2008). Exposure in vivo versus operant graded activity in chronic low back pain patients: Results of a randomized controlled trial. Pain, 138, 192-207. http://dx.doi.org/10 .1016/j.pain.2007.12.009

Linton, S. J. (2000). A review of psychological risk factors in back and neck pain. Spine, 25, 1148-1156. http://dx.doi.org/10.1097/00007632200005010-00017

Linton, S. J., Boersma, K., Jansson, M., Overmeer, T., Lindblom, K., \& Vlaeyen, J. W. (2008). A randomized controlled trial of exposure in vivo for patients with spinal pain reporting fear of work-related activities. European Journal of Pain, 12, 722-730. http://dx.doi.org/10.1016/j .ejpain.2007.11.001

Linton, S. J., Vlaeyen, J., \& Ostelo, R. (2002). The back pain beliefs of health care providers: Are we fear-avoidant? Journal of Occupational Rehabilitation, 12, 223-232. http://dx.doi.org/10.1023/A:1020218422974

Macedo, L. G., Smeets, R. J., Maher, C. G., Latimer, J., \& McAuley, J. H. (2010). Graded activity and graded exposure for persistent nonspecific low back pain: A systematic review. Physical Therapy, 90, 860-879. http://dx.doi.org/10.2522/ptj.20090303

McCracken, L. M., \& Dhingra, L. (2002). A short version of the Pain anxiety symptoms scale (Pass-20): Preliminary development and validity. Pain Research and Management, 7, 45-50. http://dx.doi.org/10 1155/2002/517163

Mewes, R., Rief, W., Stenzel, N., Glaesmer, H., Martin, A., \& Brähler, E. (2009). What is "normal" disability? An investigation of disability in the general population. Pain, 142, 36-41. http://dx.doi.org/10.1016/j.pain .2008.11.007

Meyer, K., Sprott, H., \& Mannion, A. F. (2008). Cross-cultural adaptation, reliability, and validity of the German version of the Pain Catastrophizing Scale. Journal of Psychosomatic Research, 64, 469-478. http:// dx.doi.org/10.1016/j.jpsychores.2007.12.004

Morley, S., Eccleston, C., \& Williams, A. (1999). Systematic review and meta-analysis of randomized controlled trials of cognitive behaviour therapy and behaviour therapy for chronic pain in adults, 
excluding headache. Pain, 80, 1-13. http://dx.doi.org/10.1016/S03043959(98)00255-3

Nagel, B., Gerbershagen, H. U., Lindena, G., \& Pfingsten, M. (2002). Entwicklung und empirische Uberprüfung des Deutschen Schmerzfragebogens der DGSS [Development and evaluation of the multidimensional German pain questionnaire]. Schmerz (Berlin, Germany), 16, 263-270. http://dx.doi.org/10.1007/s00482-002-0162-1

O’Sullivan, K., Dankaerts, W., O’Sullivan, L., \& O'Sullivan, P. B. (2015). Cognitive functional therapy for disabling nonspecific chronic low back pain: Multiple case-cohort study. Physical Therapy, 95, 1478-1488. http://dx.doi.org/10.2522/ptj.20140406

Pollard, C. A. (1984). Preliminary validity study of the pain disability index. Perceptual and Motor Skills, 59, 974. http://dx.doi.org/10.2466/ pms.1984.59.3.974

Qaseem, A., Wilt, T. J., McLean, R. M., \& Forciea, M. A. (2017). Noninvasive treatments for acute, subacute, and chronic low back pain: A clinical practice guideline from the American College of Physicians. Annals of Internal Medicine, 166, 514-530. http://dx.doi.org/10.7326/ M16-2367

Riecke, J., Holzapfel, S., Rief, W., \& Glombiewski, J. A. (2013). Evaluation and implementation of graded in vivo exposure for chronic low back pain in a German outpatient setting: A study protocol of a randomized controlled trial. Trials, 14, 203. http://dx.doi.org/10.1186/17456215-14-203

Riecke, J., Holzapfel, S., Rief, W., Lachnit, H., \& Glombiewski, J. A. (2016). Cross-cultural adaption of the German Quebec Back Pain Disability Scale: An exposure-specific measurement for back pain patients. Journal of Pain Research, 9, 9-15. http://dx.doi.org/10.2147/JPR .S92615

Roelofs, J., McCracken, L., Peters, M. L., Crombez, G., van Breukelen, G., \& Vlaeyen, J. (2004). Psychometric evaluation of the Pain Anxiety Symptoms Scale (PASS) in chronic pain patients. Journal of Behavioral Medicine, 27, 167-183. http://dx.doi.org/10.1023/B:JOBM.0000019850 $.51400 . \mathrm{a} 6$

Rusu, A. C., Kreddig, N., Hallner, D., Hülsebusch, J., \& Hasenbring, M. I. (2014). Fear of movement/(Re)injury in low back pain: Confirmatory validation of a German version of the Tampa Scale for Kinesiophobia. BMC Musculoskeletal Disorders, 15, 280. http://dx.doi.org/10.1186/ 1471-2474-15-280

Sehgal, N., Colson, J., \& Smith, H. S. (2013). Chronic pain treatment with opioid analgesics: Benefits versus harms of long-term therapy. Expert Review of Neurotherapeutics, 13, 1201-1220. http://dx.doi.org/10.1586/ 14737175.2013.846517

Soer, R., Köke, A. J. A., Vroomen, P. C., Stegeman, P., Smeets, R. J., Coppes, M. H., \& Reneman, M. F. (2013). Extensive validation of the pain disability index in 3 groups of patients with musculoskeletal pain. Spine, 38, E562E568. http://dx.doi.org/10.1097/BRS.0b013e31828af21f

Sullivan, M. J., Bishop, S. R., \& Pivik, J. (1995). The pain catastrophizing scale: Development and validation. Psychological Assessment, 7, 524532. http://dx.doi.org/10.1037/1040-3590.7.4.524
Tait, R. C., \& Chibnall, J. T. (2005). Factor structure of the pain disability index in workers compensation claimants with low back injuries. Archives of Physical Medicine and Rehabilitation, 86, 1141-1146. http:// dx.doi.org/10.1016/j.apmr.2004.11.030

Trost, Z., France, C. R., \& Thomas, J. S. (2009). Examination of the photograph series of daily activities (PHODA) scale in chronic low back pain patients with high and low kinesiophobia. Pain, 141, 276-282. http://dx.doi.org/10.1016/j.pain.2008.11.016

Turk, D. C. (2003). Cognitive-behavioral approach to the treatment of chronic pain patients. Regional Anesthesia and Pain Medicine, 28, 573-579. http://dx.doi.org/10.1097/00115550-200311000-00016

Underwood, M., \& Buchbinder, R. (2013). Red flags for back pain. British Medical Journal, 347, f7432. http://dx.doi.org/10.1136/bmj.f7432

Van Tulder, M., \& Koes, B. (2006). Chronic low back pain. American Family Physician, 74, 1577-1579.

Vibe Fersum, K., O’Sullivan, P., Skouen, J. S., Smith, A., \& Kvåle, A. (2013). Efficacy of classification-based cognitive functional therapy in patients with non-specific chronic low back pain: A randomized controlled trial. European Journal of Pain, 17, 916-928. http://dx.doi.org/ 10.1002/j.1532-2149.2012.00252.x

Vlaeyen, J. W., Crombez, G., \& Linton, S. J. (2009). The fear-avoidance model of pain. Pain, 157, 1588-1589. http://dx.doi.org/10.1097/j.pain .0000000000000574

Vlaeyen, J. W., de Jong, J., Geilen, M., Heuts, P. H., \& van Breukelen, G. (2001). Graded exposure in vivo in the treatment of pain-related fear: A replicated single-case experimental design in four patients with chronic low back pain. Behaviour Research and Therapy, 39, 151-166. http:// dx.doi.org/10.1016/S0005-7967(99)00174-6

Vlaeyen, J., Morley, S., Linton, S., Boersma, K., \& de Jong, J. (2012) Pain-related fear: Exposure-based treatment for chronic pain. Seattle, WA: IASP Press.

Wicksell, R. K., Lekander, M., Sorjonen, K., \& Olsson, G. L. (2010). The Psychological Inflexibility in Pain Scale (PIPS)—Statistical properties and model fit of an instrument to assess change processes in pain related disability. European Journal of Pain, 14, 771.e1-e14. http://dx.doi.org/ 10.1016/j.ejpain.2009.11.015

Williams, A. C. D., Eccleston, C., \& Morley, S. (2012). Psychological therapies for the management of chronic pain (excluding headache) in adults. Cochrane Database of Systematic Reviews, 11, CD007407. http://dx.doi.org/10.1002/14651858.CD007407.pub3

Woods, M. P., \& Asmundson, G. J. G. (2008). Evaluating the efficacy of graded in vivo exposure for the treatment of fear in patients with chronic back pain: A randomized controlled clinical trial. Pain, 136, 271-280. http://dx.doi.org/10.1016/j.pain.2007.06.037

Received September 4, 2017

Revision received January 30, 2018 Accepted January 30, 2018 